TITLE:

\title{
Shape design of curved surface of membrane structure using developable surface
}

$\operatorname{AUTHOR}(\mathrm{S}):$

Cui, Jinglan; Ohsaki, Makoto

\section{CITATION:}

Cui, Jinglan ...[et al]. Shape design of curved surface of membrane structure using developable surface. Journal of the International Association for Shell and Spatial Structures 2018, 59(3): 199-214

ISSUE DATE:

2018-09-01

URL:

http://hdl.handle.net/2433/236070

RIGHT:

許諾条件に基づいて掲載しています。The full-text file will be made open to the public on 01 September 2019 in accordance with publisher's 'Terms and Conditions for Self-Archiving'. 


\title{
SHAPE DESIGN OF CURVED SURFACE OF MEMBRANE STRUCTURE USING DEVELOPABLE SURFACE
}

\author{
Jinglan CUI ${ }^{1}$ and Makoto OHSAKI ${ }^{2}$ \\ ${ }^{1}$ Dept. of Architecture and Architectural Engineering, Kyoto University, Japan, \\ cui.jinglan.63w@st.kyoto-u.ac.jp \\ ${ }^{2}$ Dept. of Architecture and Architectural Engineering, Kyoto University, Japan, \\ ohsaki@archi.kyoto-u.ac.jp
}

Editor's Note: Manuscript submitted 9 January 2018; revision received 2 August; accepted 7 September 2018. This paper is open for written discussion, which should be submitted to the IASS Secretariat no later than March 2019.

DOI: https://doi.org/10.20898/j.iass.2018.197.005

\section{ABSTRACT}

A method is presented for design of curved surface of frame supported membrane structures. Since a uniform stress state is desired, the minimal surface is often chosen as an ideal target curved surface. Another ideal shape is a developable surface that can be generated from a plane sheet. Therefore, in this paper, the minimal surface, the developable surface, and an intermediate surface between them are chosen as the target curved surface. The cutting patterns are generated by reducing the uniform stress from the plane sheet obtained from the curved surface, where correction coefficients are incorporated. In the numerical examples, the properties of selfequilibrium shape and its stress distribution are investigated for surfaces generated from the three kinds of target curved surfaces.

Keywords: developable surface, minimal surface, membrane structure, cutting pattern

\section{INTRODUCTION}

Membrane structures used for covering large space in architectural and civil engineering are stabilized by introducing tension into a soft membrane material that can resist external loads only by tensile stress. Membrane roofs are aesthetically efficient because of their lightweight and transparent properties. However, the membrane material cannot have compressive force, out-of-plane shear force, and outof-plane bending moment; accordingly, wrinkling easily occurs due to stress concentration and nonuniform stress distribution. Therefore, a process called form finding is needed to find a curved surface shape that can be at equilibrium with tensile membrane force.

The approaches to form finding is classified into simulation of hanging models, numerical simulation of soap films, and structural shape optimization [1]. Beatini and Royer-Carfagni [2] presented soap film analogy method for membrane with different stresses in two orthogonal directions. Bletzinger et al. [3] proposed a framework of shape optimization based on the updated reference strategy for analysis and parametric modeling of surface developed in the field of computer-aided geometric design (CAGD) [4].

Since the membrane structure can be realized by connecting several planar sheets, it is desirable to design the cutting pattern so that uniform in-plane tension stress is generated in the membrane. The most conventional approach utilizes the geodesic line [5] to obtain approximate cutting patterns from the target curved surface [6, 7]. Punurai et al. [8] proposed an optimization approach using genetic algorithm. Gale and Lewis [9] proposed a discrete element model using dynamic relaxation [10] and a method of forming a tensile fabric structures.

Many methods have been proposed for design and optimization of cutting pattern including those in the fields of computational geometry and design of fabrics for clothes; however, those methods generally need to carry out geometrically nonlinear analysis for finding the self-equilibrium shape at each step of optimization, which is computationally expensive [11]. Therefore, it is important to present a simple method for obtaining a cutting pattern [12] 
that can lead to an equilibrium shape close to the target shape with uniform tension state. Tsubota et al. [13] proposed a method for adjusting the boundary shape of cutting patterns so that equilibrium shape generated by connecting the membrane sheets to the specified boundary has uniform tension state. Ohsaki et al. [14, 15] formulated an inverse problem for shape design of cutting patterns, and proposed an optimization method for generating cutting patterns so that the stresses at equilibrium of membrane discretized into triangular finite elements are close to the specified target values. Shon et al. [16] proposed a method for adjusting smooth spline approximation of the geodesic lines on the subplane and generated smooth cutting patterns. Moncrieff and Topping [17] proposed a method of generating a cutting pattern using the geometrical unfolding method and least squares minimizing procedure based on the dynamic relaxation method and the force density method.

In the conventional design method of the membrane structures [6], a minimal surface [18] that is equivalent to a uniform stress surface is considered as an ideal target curved surface. Yokosuka and Honma [19] proposed a numerical computation procedure to solve the minimal surface problem by using mean curvature based on discrete differential geometry. Gosling and Lewis [20] proposed a method for finding a minimal surface using quadrilateral finite elements and dynamic relaxation method. However, a difficulty arises from the fact that the membrane surface is generated by stretching and connecting plane membrane sheets; therefore, it is desirable that the surface is close to a developable surface rather than the minimal surface. By contrast, a developable surface cannot have uniform tension state. Therefore, intermediate surface between the developable surface and minimal surface seems to be an optimal solution as a target surface for designing membrane structures. Developable surfaces are extensively studied in the field of CAGD for approximation of curved surfaces using parametric representations [21, 22]. Cui et al. [23] and Nakamura et al. [24] proposed a method for optimizing the shape of a free-form shell modeled using the $(1, n)$-Bézier surface. However, effectiveness of using the developable surface has not been well discussed in the existing literatures.

In this paper, we present a method for designing curved surface of frame-supported membrane structures. The target curved surface is modeled as a developable surface, the minimal surface, or an intermediate surface between them, which is obtained by solving a multiobjective programming problem. The cutting pattern is generated by removing the target ideal stress from the selfequilibrium state, where a correction coefficient is introduced to improve the average stress in each direction. Performance of the proposed method is verified using a small membrane model, and an example of complex membrane structure obtained by connecting simple surface is presented to show applicability of the method to a practical problem.

\section{FORMULATION OF TARGET CURVED SURFACE}

\subsection{Formulation of developable surface}

In the process of shape design of membrane structures, a target curved surface is first specified by a designer or an architect. The surface is represented by a Bézier surface, and the coordinates of its control points are used as variables. The developable surface can be defined as a curved surface where the Gaussian curvature vanishes everywhere on the surface, and it can also be defined as a curved surface with a straight generating line without torsion [21]. A minimal surface such as the hyperbolic paraboloid (HP) surface, which is classified as ruled surface, also has a straight generating line. Therefore, the $(1, n)$-Bézier surface that has a straight line in one direction is used here for modeling the target curved surface. The formulation for developable surface is described below for completeness of the paper. See Refs. [22-24] for details.

A developable surface is defined by assigning a developability condition [25] to the ruled surface. Denoting the directing curves by $\mathbf{f}(u)$ and $\mathbf{h}(u)$, the ruled surface $\mathbf{s}(u, v)$ is given by the following equation:

$$
\mathbf{s}(u, v)=(1-v) \mathbf{f}(u)+v \mathbf{h}(u)
$$

where, $u$ and $v$ are the parameters that vary from 0 to 1.

Both of $\mathbf{h}(u)$ and $\mathbf{f}(u)$ are defined by the parameter $u$, and the line segment connecting the two points of the same parameter value of $\mathbf{f}(u)$ and $\mathbf{h}(u)$ becomes a generating line. Fig. 1 shows a developable surface that is a ruled surface satisfying the developability condition. The vectors $\mathbf{s}_{u}(u, v)$ 
and $\mathbf{n}_{s}(u, v)$ are the tangent vector in the direction of parameter $u$ and the normal vector at $\mathbf{s}(u, v)$, respectively. In addition, $\mathbf{n}_{f}(u)$ and $\mathbf{n}_{h}(v)$ represent the normal vectors of the curved surface along the boundaries defined by $\mathbf{f}(u)$ and $\mathbf{h}(u)$, respectively. Since the generating line of a developable surface does not have torsion, the tangent vector $\mathbf{s}_{u}$ at an arbitrary point on the generating line and the direction vector $\mathbf{h}(u)-\mathbf{f}(u)$ of the generating line are in the same plane as illustrated in Fig. 1. Similarly, the normal vector $\mathbf{n}_{s}(u, v)$ and generating line are in the same plane. Let $\mathbf{f}_{u}(u)$ and $\mathbf{h}_{u}(v)$ denote the tangent vectors of $\mathbf{f}(u)$ and $\mathbf{h}(u)$. The condition that the ruled surface defined by Eq. (1) becomes a developable surface is stated that the tangent vectors of directing curves and the direction vector of generating line are in the same plane, which can be expressed as follows by using a scalar triple product [26]:

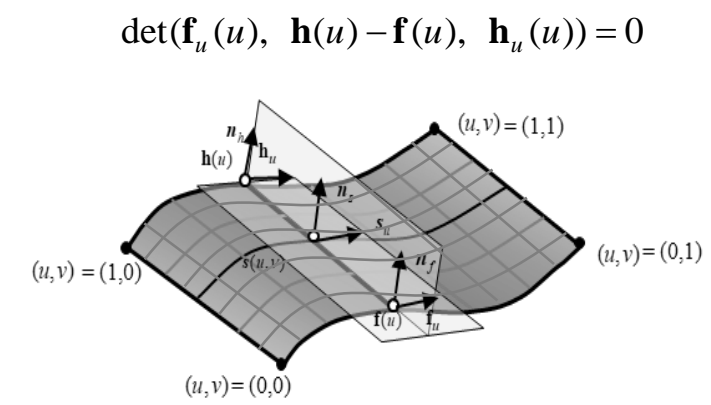

Figure 1: A developable surface defined as a ruled surface satisfying developability condition

The directing curves $\mathbf{f}(u)$ and $\mathbf{h}(u)$ are defined as $n$th order Bézier curves defined as

$$
\mathbf{P}^{n}(u)=\sum_{i=0}^{n} \mathbf{Q}_{i} B_{i}^{n}(u)
$$

where $\mathbf{Q}_{i}=\left(q_{x}^{i}, q_{y}^{i}, q_{z}^{i}\right)^{T}$ is the coordinate vector of the $i$ th control point, and $B_{i}^{n}(u)$ is the $n$th order Bernstein basis function defined by

$$
B_{i}^{n}(u)=\frac{n !}{i !(n-i) !} u^{i}(1-u)^{n-i}
$$

The control points of $\mathbf{f}(u)$ and $\mathbf{h}(u)$ are denoted by $\mathbf{Q}_{i}^{f}$ and $\mathbf{Q}_{i}^{h}$, respectively, as

$$
\begin{aligned}
& \mathbf{f}(u)=\sum_{i=0}^{n} \mathbf{Q}_{i}^{f} B_{i}^{n}(u) \\
& \mathbf{h}(u)=\sum_{i=0}^{n} \mathbf{Q}_{i}^{h} B_{i}^{n}(u)
\end{aligned}
$$

By substituting Eqs. (5) and (6) into Eq. (1), the surface can be defined as a $(1, n)$-Bézier surface as

$$
\mathbf{s}(u, v)=(1-v) \sum_{i=0}^{n} \mathbf{Q}_{i}^{f} B_{i}^{n}(u)+v \sum_{i=0}^{n} \mathbf{Q}_{i}^{h} B_{i}^{n}(u)
$$

Figure 2: A developable surface defined by a Bézier surface

An example of $(1, n)$-Bézier surface $(n=3)$ is shown in Fig. 2. The target curved surface is modeled by connecting several $(1, n)$-Bézier surfaces. Let $\mathbf{Q}$ denote the vector consisting of variable components of $\mathbf{Q}_{i}^{f}$ and $\mathbf{Q}_{i}^{h}$ of all surfaces. Using the de Castelau's algorithm, the developability condition (2) can be formulated as a polynomial of order 3(n-1) with respect to $\mathbf{Q}$. Therefore, the condition

(2) should be satisfied at $n_{d}=3(n-1)+1=3 n-2$ different values of parameter $u$ of each surface; i.e., The developability conditions are written in the following form:

$$
\begin{aligned}
& D_{i j}(\mathbf{Q})=\operatorname{det}\left(\mathbf{f}_{u}\left(u_{j}\right), \mathbf{h}\left(u_{j}\right)-\mathbf{f}\left(u_{j}\right), \mathbf{h}_{u}\left(u_{j}\right)\right)=0 \\
& \left(i=1, \ldots, m_{s} ; j=1, \ldots n_{d}\right)
\end{aligned}
$$

where $m_{s}$ is the number of surfaces. See Refs. [23, 24, 26, 27] for details.

Continuity conditions between surfaces are classified into $G^{0}$, which ensure continuity of the nodal locations, and $G^{1}$, which also ensures the continuity of unit normal vector of surface. These conditions are represented by 


$$
S_{j}(\mathbf{Q})=0(j=1, \ldots, s)
$$

where $s$ is the number of connecting lines between surfaces. Then, the following optimization problem is to be solved:

$$
\begin{cases}\min _{\mathbf{Q}} . & D(\mathbf{Q})=\sum_{i=1}^{m_{s}} \sum_{j=1}^{n_{d}}\left(D_{i j}(\mathbf{Q})\right)^{2} \\ \text { s.t. } & S_{j}(\mathbf{Q})=0, \quad(j=1, \cdots \cdots s) \\ & \mathbf{Q}^{L} \leq \mathbf{Q} \leq \mathbf{Q}^{U}\end{cases}
$$

where $\mathbf{Q}^{U}$ and $\mathbf{Q}^{L}$ are the vectors of upper and lower bounds of $\mathbf{Q}$ representing the allowable range of control points.

Since the developable surface is a curvilinear surface that can be developed to a plane, it is suitable as a target curved surface of a membrane structure that is generated from plane cutting sheets. However, since the Gaussian curvature of a developable surface vanishes everywhere on the surface, it cannot be an equilibrium shape of the membrane structure in the tension state.

\subsection{Formulation of a minimal surface}

A minimal surface is defined as a surface that minimizes the area of surface for the specified boundary shape. It is known that the mean curvature of a minimal surface vanishes everywhere on the surface [28]. On the other hand, the mean curvature of the equilibrium shape of a membrane structure with uniform tension vanishes everywhere on the surface [29]. Therefore, the minimal surface such as the HP surface, which has straight generating lines and negative Gaussian curvatures at any point on the surface, is usually used as the target curved surface of a membrane structure.

The parameter space $(u, v)$ of each Bézier surface is divided into $n_{g}=n_{u} \times n_{v}$ grids with the size $1 / n_{u}$ and $1 / n_{v}$, respectively, in $u$ - and $v$-directions, as shown in the grids in Fig. 1. Let $\kappa_{i j 1}(\mathbf{Q})$ and $\kappa_{i j 2}(\mathbf{Q})$ denote the principal curvatures evaluated at the center of the $j$ th grid of the $i$ th surface. The mean curvature $H_{i j}(\mathbf{Q})$ is computed as

$$
H_{i j}(\mathbf{Q})=\frac{\kappa_{i j 1}(\mathbf{Q})+\kappa_{i j 2}(\mathbf{Q})}{2},\left(i=1, \ldots, m_{s} ; j=1, \ldots n_{g}\right)
$$

See, e.g., Ref. [30] for detailed derivation of the curvatures of a Bézier surface. A shape close to a minimal surface is obtained by solving the following optimization problem for minimizing the sum of squares of $H_{i j}(\mathbf{Q})$ of the $(1, n)$-Bézier surface:

$$
\begin{cases}\min _{\mathbf{Q}} . & H(\mathbf{Q})=\sum_{i=1}^{m_{s}} \sum_{j=1}^{n_{g}}\left(H_{i j}(\mathbf{Q})\right)^{2} \\ \text { s.t. } & S_{j}(\mathbf{Q})=0, \quad(j=1, \cdots \cdots s) \\ & \mathbf{Q}^{L} \leq \mathbf{Q} \leq \mathbf{Q}^{U}\end{cases}
$$

Although a uniform tension state is achieved by a minimal surface, it cannot be generated from a plane membrane sheet. By contrast, a developable surface is generated from a plane sheet; however, the stresses of an equilibrium shape of developable surface cannot be uniform. In order to incorporate the merits of both developability and uniform tension state, we use the constraint method of multiobjective optimization for finding trade-off values of $D(\mathbf{Q})$ and $H(\mathbf{Q})$.

Let $D^{*}(\mathbf{Q})$ and $\hat{H}(\mathbf{Q})$ denote the values of $D(\mathbf{Q})$ and $H(\mathbf{Q})$, respectively, of the optimal solution of problem (10) for minimizing $D(\mathbf{Q})$. The values of $D(\mathbf{Q})$ and $H(\mathbf{Q})$ of the optimal solution of problem (12) for minimizing $H(\mathbf{Q})$ are denoted by $\hat{D}(\mathbf{Q})$ and $H^{*}(\mathbf{Q})$, respectively. In view of these values, the upper bound $\bar{H}(\mathbf{Q})$ for $H(\mathbf{Q})$ is specified as the value between $\hat{H}(\mathbf{Q})$ and $H^{*}(\mathbf{Q})$. Then, the following single-objective optimization problem is solved to find a surface having intermediate characteristics between the developable surface and the minimal surface:

$$
\begin{cases}\underset{\mathbf{Q}}{\min } . & D(\mathbf{Q}) \\ \text { s.t. } & H(\mathbf{Q}) \leq \bar{H}(\mathbf{Q}) \\ & S_{j}(\mathbf{Q})=0, \quad(j=1, \cdots \cdots s) \\ & \mathbf{Q}^{L} \leq \mathbf{Q} \leq \mathbf{Q}^{U}\end{cases}
$$

The value of $\bar{H}(\mathbf{Q})$ is first determined as the mean value $\left(\hat{H}(\mathbf{Q})+H^{*}(\mathbf{Q})\right) / 2$ of $\hat{H}(\mathbf{Q})$ and $H^{*}(\mathbf{Q})$, and the optimization problem (13) is solved. Then, if the stress distribution is not acceptable, we reduce the value $\bar{H}(\mathbf{Q})$ to be close to $\hat{H}(\mathbf{Q})$. We can further modify the value of $\bar{H}(\mathbf{Q})$ using the bi-section approach. 


\section{DETERMINATION OF CUTTING PATTERN AND EQUILIBRIUM SHAPE}

\subsection{Projection of target curved surface to a plane}

When designing the cutting pattern, it is important to develop the target curved surface with high precision on the plane. Ohsaki et al. [31, 32] presented an iterative approach to obtain an approximate optimal shape of cutting pattern by projecting the curved surface of equilibrium to a plane. In their method, the stress is removed from the shape at equilibrium to obtain a cutting sheet. Here, we first project the target curved surface without stress to a plane. The stresses are removed later as described in Sec. 3.2. Outline of the method is as follows, which is illustrated in Fig. 3:

Step 1: Divide the target curved surface into triangular elements.

Step 2: Project the triangulated surface to a plane.

Step 3: Minimize the difference between the shapes of triangle on the curved surface and triangle on the plane to obtain optimal development to the plane.

When optimizing the shape of the cutting pattern, $(x, y)$-coordinates of all the nodes of triangles on the plane are used as design variables, while the two components of rigid body displacement as well as rigid body rotation are fixed. Denote the lengths of three edges of the $i$ th triangle on the target curved surface by $l_{i 1}^{0}, l_{i 2}^{0}$ and $l_{i 3}^{0}$, respectively. The corresponding lengths of the triangle on the plane are denoted by $l_{i 1}, l_{i 2}$ and $l_{i 3}$, respectively. Let $\mathbf{P}$ denote the vector consisting of the variable components of the coordinates $\left(x_{k}, y_{k}\right)$ of all nodes. When the target curved surface consists of $n_{c}$ variable nodes and $n_{e}$ elements, the optimization problem is formulated as

$$
\left\{\begin{array}{ll}
\min _{\mathbf{P}} & F(\mathbf{P})=\sum_{i=1}^{n_{e}} \sum_{j=1}^{3}\left(l_{i j}(\mathbf{P})-l_{i j}^{0}\right)^{2} \\
\text { s.t. } \quad & x_{k}^{L} \leq x_{k} \leq x_{k}^{U} \\
& y_{k}^{L} \leq y_{k} \leq y_{k}^{U}
\end{array} \quad\left(k=1,2, \ldots, n_{c}\right)\right.
$$

where $\left(x_{k}^{U}, y_{k}^{U}\right)$ and $\left(x_{k}^{L}, y_{k}^{L}\right)$ are the upper and lower bounds of $\left(x_{k}, y_{k}\right)$, which are assigned so that the surface remains in on the plan specified by the initial shape. When the curvature of target curved surface is small, it can be linearly projected on a plane. However, if the curvature is large, it is effective to project it in the direction of the center of curvature. See Refs. [31, 32] for details.

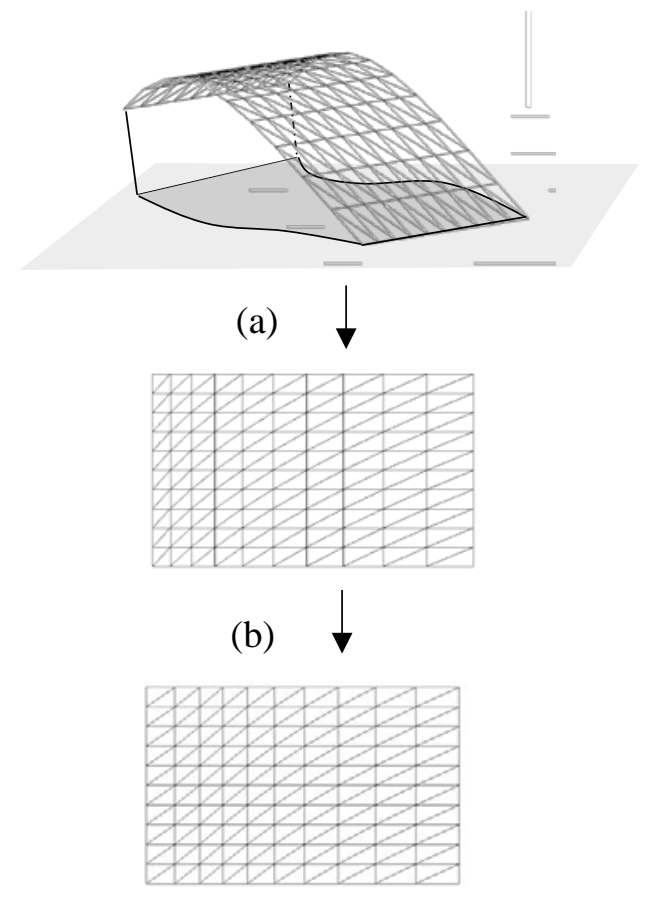

(c)

Figure3: Illustration of development process to a plane: (a) target curved surface, (b) projected surface (c) shape after minimization of error

\subsection{Generation of cutting pattern}

An approximate plane cutting sheet for covering the target curved surface is obtained using the procedure in Sec. 3.1. However, in this process, the required tension forces in the surface are not incorporated. Here, the surface developed to a plane is shrunk so that the desired membrane stress is generated after connecting the cutting sheet to the boundary of the surface.

The membrane material is modeled as an elastic orthotropic material. In the following equations, the local coordinates are assumed to coincide with the principal directions for simple presentation of equations, although an appropriate coordinate transformation is applied in the numerical examples. Let $E_{x}$ and $E_{y}$ denote the elastic moduli in the warp and weft directions satisfying $E_{x} \geq E_{y}$. The shear 
modulus and Poisson's ratios are denoted by $G, v_{x y}$ and $v_{y x}$, respectively. The stresses in $x$ - and $y$ directions and shear stress are denoted by $\sigma_{x}^{e}, \sigma_{y}^{e}$ and $\tau_{x y}^{e}$, respectively. The strains are also denoted by $\varepsilon_{x}^{e}, \varepsilon_{y}^{e}$ and $\gamma_{x y}^{e}$. Using the symbols $\eta=E_{x} / E_{y}$ and $\kappa=G / E_{y}$, the following relation holds between the vectors of element stress $\boldsymbol{\sigma}=\left(\sigma_{x}^{e}, \sigma_{y}^{e}, \tau_{x y}^{e}\right)^{T}$ and the element strain $\boldsymbol{\varepsilon}=\left(\varepsilon_{x}^{e}, \varepsilon_{y}^{e}, \gamma_{x y}^{e}\right)^{T}$ with respect to the stress-strain relation matrix $\mathbf{D}$ :

$$
\begin{gathered}
\boldsymbol{\sigma}=\mathbf{D} \boldsymbol{\varepsilon} \\
\mathbf{D}=\frac{E_{y}}{1-\eta v_{x y}^{2}}\left[\begin{array}{ccc}
\eta & \eta v_{x y} & 0 \\
\eta v_{x y} & 1 & 0 \\
0 & 0 & \kappa\left(1-\eta v_{x y}^{2}\right)
\end{array}\right]
\end{gathered}
$$

Assigning the desired uniform stress $\boldsymbol{\sigma}_{0}=\left(\sigma_{x}^{0}, \sigma_{y}^{0}, 0\right)^{T}$ to the target curved surface, the uniform strain $\varepsilon_{0}=\left(\varepsilon_{x}^{0}, \varepsilon_{y}^{0}, 0\right)^{T}$ is determined using Eq. (15). As a result, the contraction ratios in the two principal directions are obtained, and the cutting pattern is updated by the following equation:

$$
\left\{\begin{array}{l}
\mathbf{x}_{\mathrm{p}}=\mathbf{x}\left(1-\varepsilon_{x}^{0}\right) \\
\mathbf{y}_{\mathrm{p}}=\mathbf{y}\left(1-\varepsilon_{y}^{0}\right)
\end{array}\right.
$$

where, $\mathbf{x}_{\mathrm{p}}$ and $\mathbf{y}_{\mathrm{p}}$ are the nodal coordinate vectors of the cutting pattern in the unstressed state, and $\mathbf{x}$ and $\mathbf{y}$ are the nodal coordinate vectors obtained by solving the problem (14).

The cutting pattern generated by Eq. (17) is attached to the boundary frame to obtain the curved selfequilibrium shape. This process is formulated as a forced displacement analysis problem without external force. We solve this problem by minimizing the total strain energy. See Ref. [31, 32, 33] for details. It has been confirmed that the number of mesh division in the following each model is enough to express the properties of the membrane surface.

\section{PRELIMINARY INVESTIGATION AND CORRECTION OF PROPOSED METHOD}

\subsection{Investigation of cutting pattern generation method}

Cutting patterns and equilibrium shapes can be found using the method described in Secs. 2 and 3.
However, the stress of the obtained membrane structure does not coincide with the desired uniform stress. Accuracy of the cutting pattern is investigated below using a simple model of developable surface.

We generate a target curved surface as shown in Fig. 4(a) on a $6 \times 4$ m rectangular plan. Fig. 4(b) shows an example of a developable surface. It is expected that the membrane surface is designed by connecting several small Bézier surfaces. Therefore, we assume each unit surface is small enough and is generated from a single cutting pattern. It is easily expected that the stress distribution is much improved if several cutting patterns are used. Therefore, a kind of extreme case is considered to investigate the performance of the proposed method. It is possible to incorporate flexibility of the boundary frame; however, we assume rigid boundary for simple presentation of the method. The membrane material is PolyVinyl Chloride (PVC) with elastic orthotropic property as $E_{x}=384 \mathrm{kN} / \mathrm{m}, \quad E_{y}=338 \mathrm{kN} / \mathrm{m}$, and Poisson's ratios $v_{x y}=0.30$ and $v_{y x}=0.34$. The target stresses are $3.0 \mathrm{kN} / \mathrm{m}$ in both of $x$ - and $y$-directions. These values are used in all of the following examples.

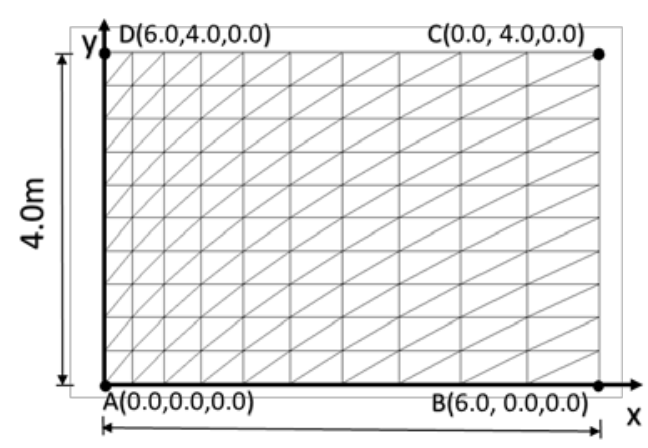

(a)

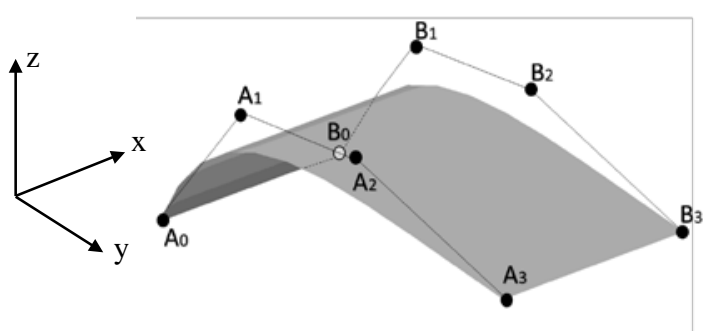

(b)

Figure 4: An example of simple developable surface: (a) plan view, (b) diagonal view 

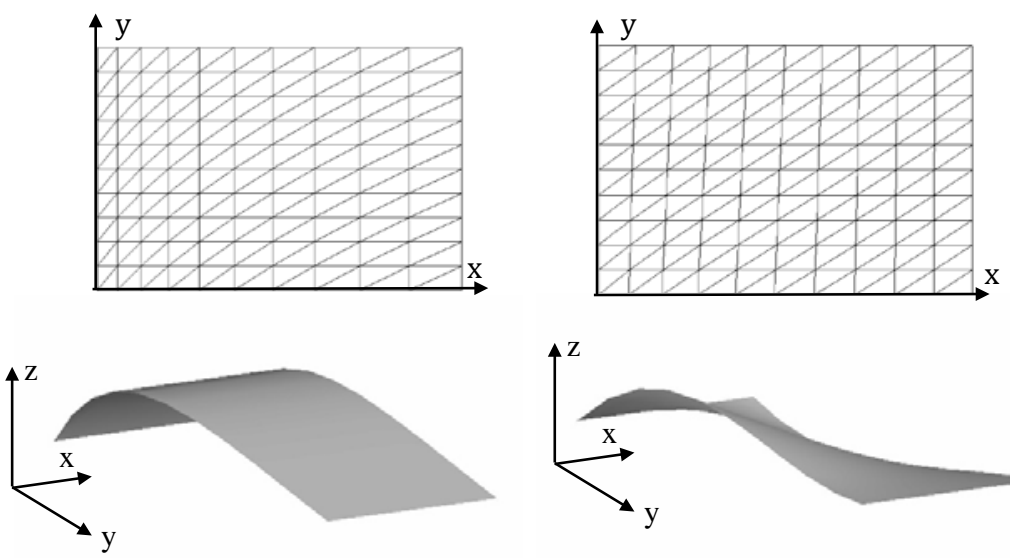

(a)

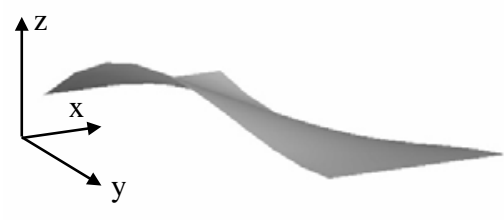

(b)
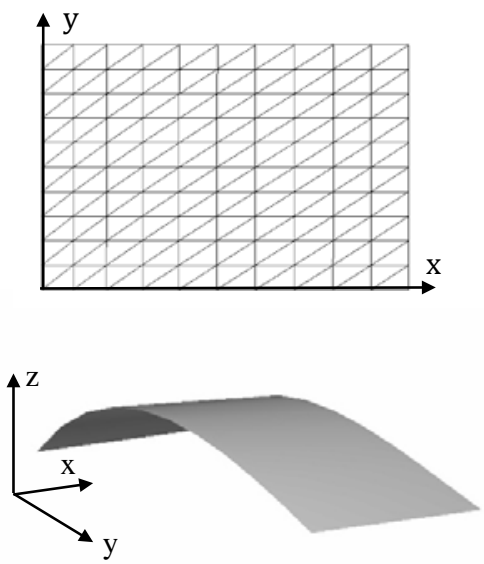

(c)

Figure 5: Plan view and diagonal view of target curved surfaces: (a) developable surface, (b) minimal surface, (c) intermediate surface between developable and minimal surfaces

As shown in Fig. 4(b), both directing curves in $x$ direction are represented by Bézier curves of order three using the control points $A_{0} \sim A_{3}$ and $B_{0} \sim$ $B_{3}$. The $x$-coordinates of $A_{1}, A_{2}, B_{1}, B_{2}$ and the $z$ coordinates of $B_{1}$ and $B_{2}$ are considered as variables in the optimization problems (10), (12) and (13). Fig. 5(a), (b) and (c) are the plan and diagonal views of the developable surface, the minimal surface and an intermediate surface between them, respectively, obtained by solving problems (10), (12) and (13). The values of $D^{*}(\mathbf{Q})$ and $\hat{H}(\mathbf{Q})$ of the developable surface are $0.000 \mathrm{~m}^{6}$ and $0.024 \mathrm{rad}^{2} / \mathrm{m}^{2}$, and $\hat{D}(\mathbf{Q})$ and $H^{*}(\mathbf{Q})$ of the minimal surface are $8.090 \times 10^{2}$ $\mathrm{m}^{6}$ and $3.660 \times 10^{-3} \mathrm{rad}^{2} / \mathrm{m}^{2} . \bar{H}(\mathbf{Q})$ for problem (13) is assigned as $0.011 \mathrm{rad}^{2} / \mathrm{m}^{2}$. Fig. 6(a), (b) and (c) show the shapes of cutting pattern and curved surface of self-equilibrium obtained from the target curved surfaces in Figs. 5(a), (b) and (c), respectively.

Table 1 shows the mean, maximum and minimum stresses of the membrane structures in Fig. 6 (a), (b) and (c), where the ratio of mean stress to the target stress is also listed in the last column. The mean stresses at self-equilibrium state are considerably below the target value $3.0 \mathrm{kN} / \mathrm{m}$, and the maximum stresses of developable and intermediate surfaces are close to the target value. Although it is not realistic to expect that the membrane stress is close to the target value even along the boundary or at the corner, wrinkle may occur if a stress is negative. Therefore, it is necessary to incorporate some correction to the process of generating cutting patterns from the target curved surface.
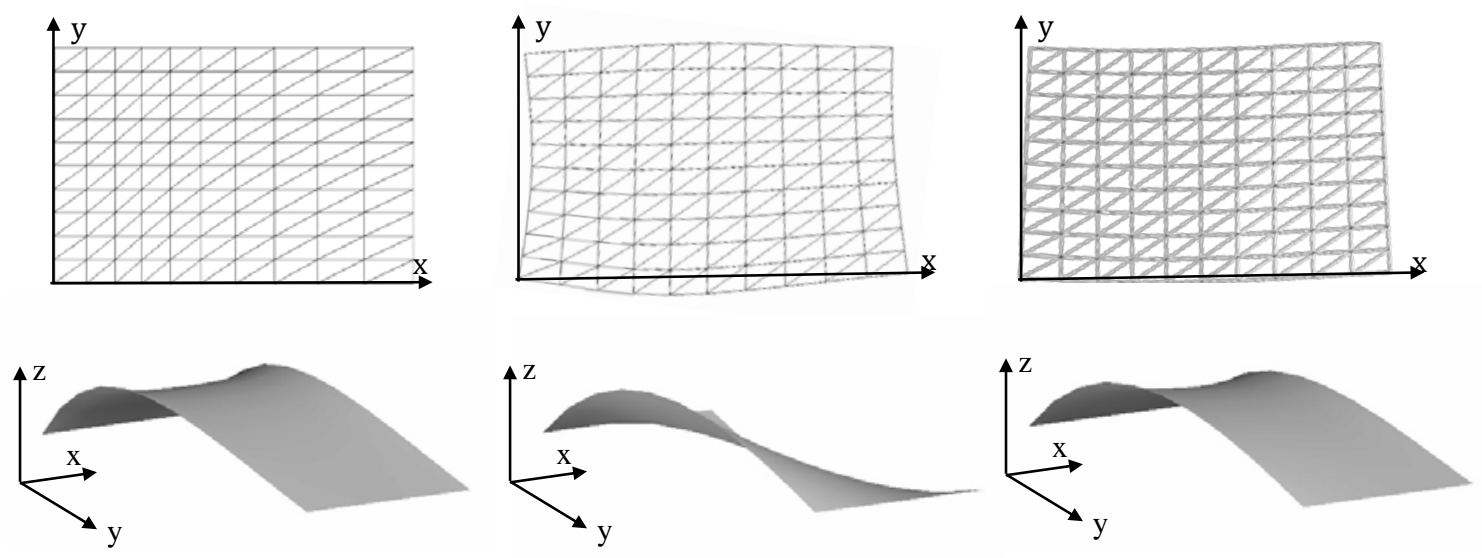

(a)

(b)

(c)

Figure 6: Cutting patterns and self-equilibrium shape generated from target curved surfaces:

(a) developable surface, (b) minimal surface, (c) intermediate surface 
Table 1: Stresses $(\mathrm{kN} / \mathrm{m})$ at self-equilibrium shapes generated from cutting patterns corresponding to three types of target curved surface

\begin{tabular}{|c|c|c|c|c|c|}
\hline \multicolumn{2}{|l|}{$\begin{array}{l}\text { Target curved } \\
\text { surface type }\end{array}$} & $\begin{array}{l}\text { Minimum } \\
\text { value }\end{array}$ & $\begin{array}{l}\text { Maximum } \\
\text { value }\end{array}$ & Mean value & Mean/target \\
\hline \multirow{2}{*}{$\begin{array}{l}\text { Developable } \\
\text { surface }\end{array}$} & $\sigma_{x}$ & 1.830 & 3.016 & 2.359 & $78.6 \%$ \\
\hline & $\sigma_{y}$ & -0.018 & 3.018 & 0.754 & $25.1 \%$ \\
\hline \multirow{2}{*}{ Minimal surface } & $\sigma_{x}$ & 0.010 & 12.232 & 2.356 & $78.5 \%$ \\
\hline & $\sigma_{y}$ & -0.388 & 6.220 & 1.814 & $60.5 \%$ \\
\hline \multirow{2}{*}{$\begin{array}{l}\text { Intermediate } \\
\text { surface }\end{array}$} & $\sigma_{x}$ & 1.972 & 3.025 & 2.405 & $80.2 \%$ \\
\hline & $\sigma_{y}$ & 0.003 & 3.611 & 0.895 & $29.8 \%$ \\
\hline
\end{tabular}

\subsection{Correction of cutting pattern generation} method

The developable surface in Fig. 5(a) with the height $Z=Z_{0}\left(Z_{0}=2 \mathrm{~m}\right)$ is scaled in $z$-direction to obtain target curved surfaces with three different heights $Z=0,0.5 Z_{0}$ and $Z_{0}$. As can be seen from Table 2, the stresses are almost uniform and coincide with the target value for a planar target surface with $Z=0$.
However, for $Z=0.5 Z_{0}$ the mean stresses in $x$ - and $y$-directions become $83.1 \%$ and $32.3 \%$, respectively, of the target value. When $Z=Z_{0}$, i.e., the target curved surface is the developable surface, the mean stresses in $x$ - and $y$-directions are $78.6 \%$ and $25.1 \%$, respectively, of the target value as shown in Table 1 . It is observed from these results that the difference from the target stress becomes larger, especially in $y$-direction, and the minimum value of the stress becomes negative as the curvature is increased.
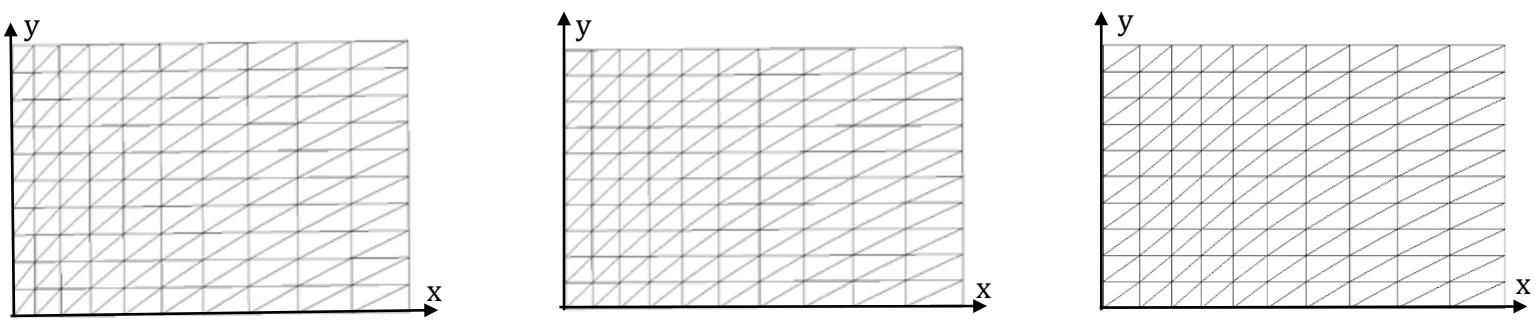

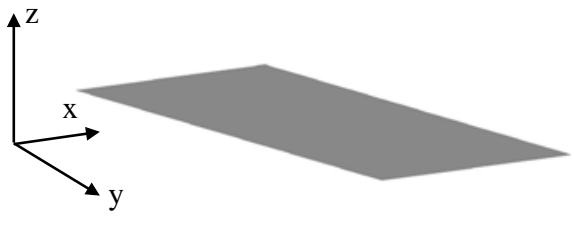

(a)

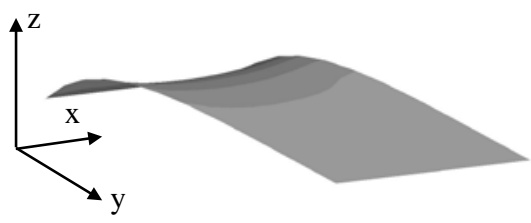

(b)

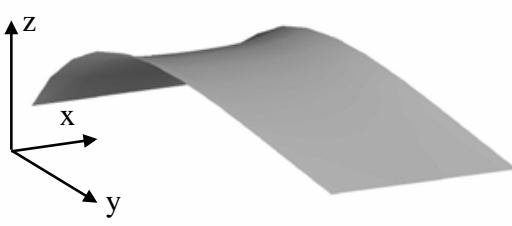

(c)

Figure 7: Cutting pattern and self-equilibrium shape obtained from developable surface scaled in z-direction: (a)

$$
Z=0 \text {, (b) } Z=0.5 Z_{0} \text {, (c) } Z=Z_{0}
$$

Table 2: Stresses $(\mathrm{kN} / \mathrm{m})$ of self-equilibrium shape obtained from developable surface scaled in z-direction

\begin{tabular}{|c|c|c|c|c|c|}
\hline Height & & Minimum value & $\begin{array}{c}\text { Maximum } \\
\text { value }\end{array}$ & Mean value & Mean/target \\
\hline \multirow{2}{*}{$\begin{array}{c}Z=0 \\
\text { (plane) }\end{array}$} & $\sigma_{x}$ & 3.015 & 3.017 & 3.016 & $100.5 \%$ \\
\hline & $\sigma_{y}$ & 3.015 & 3.019 & 3.018 & $100.6 \%$ \\
\hline \multirow{2}{*}{$Z=0.5 Z_{0}$} & $\sigma_{x}$ & 2.007 & 3.269 & 2.493 & $83.1 \%$ \\
\hline & $\sigma_{y}$ & 0.061 & 3.094 & 0.969 & $32.3 \%$ \\
\hline
\end{tabular}


To obtain the mean stress closer to the target value, Eq.(17) for generating the cutting pattern is modified as follows by introducing the reduction ratio correction coefficients $k_{x}$ and $k_{y}$ in $x$ - and $y$ directions, respectively, which are called correction coefficients for brevity:

$$
\left\{\begin{array}{l}
\boldsymbol{x}_{\mathrm{p}}=\boldsymbol{x}\left(1-k_{x} \varepsilon_{x}^{0}\right) \\
\boldsymbol{y}_{\mathrm{p}}=\boldsymbol{y}\left(1-k_{y} \varepsilon_{y}^{0}\right)
\end{array}\right.
$$

The values of $k_{x}$ and $k_{y}$ depend on the curvatures of the target curved surface, and can be decided by minimizing the deviation of the membrane stress from the target stress.

Table 3 shows the stresses at self-equilibrium for $Z=0.5 Z_{0}$ and $Z=Z_{0}$ after utilizing $k_{x}$ and $k_{y}$. The results confirm that the mean stress approaches the target value. However, the maximum stress and minimum stress are not improved. The shapes of cutting pattern and self-equilibrium state are not shown, because they are almost the same as those in Fig. 7 without any visible differences. The shape of obtained membrane structure has a slight difference with the shape of the target curved surface. The values of maximum difference $\Delta Z$ between the $Z$ coordinates at the center the surface between the selfequilibrium shape and the target surface with straight generating line are $0.172 \mathrm{~m}$ and $0.173 \mathrm{~m}$, respectively, for $Z=Z_{0}$ and $Z=0.5 Z_{0}$. The values of $\Delta Z$ are $0.046 \mathrm{~m}$ and $0.063 \mathrm{~m}$ if Eq. (17) without $k_{x}$ and $k_{y}$ is used.

It should be noted that $\Delta Z$ will be small if the developable surface is used and the target stress is 0 . The non-zero value of $\Delta Z$ is due to extension of membrane, and different values in two directions due to the use of $k_{x}$ and $k_{y}$ leads to larger values of $\Delta Z$.

Table 4 shows the stresses at self-equilibrium for the minimal surface and intermediate surface after utilizing the correction coefficients. We can confirm that the mean stress is very close to the target value. The shapes of cutting pattern and self-equilibrium state are not shown, because they are almost the same as those in Figs. 6(b), (c). The values of $\Delta Z$ are $0.074 \mathrm{~m}$ and $0.162 \mathrm{~m}$, respectively, for minimal surface and intermediate surface. Those values are $0.057 \mathrm{~m}$ and $0.050 \mathrm{~m}$ if Eq. (17) is used.

Table 3: Stresses $(\mathrm{kN} / \mathrm{m})$ of self-equilibrium shape obtained using correction coefficients from developable surface scaled in z-direction

\begin{tabular}{c|cccccc}
\hline \multicolumn{1}{c}{ Height } & & $\begin{array}{c}\text { Minimum } \\
\text { value }\end{array}$ & $\begin{array}{c}\text { Maximum } \\
\text { value }\end{array}$ & Mean value & Mean/target & $\begin{array}{c}\text { Correction } \\
\text { coefficient }\end{array}$ \\
\hline$Z=0.5 Z_{0}$ & $\sigma_{x}$ & 1.338 & 5.738 & 3.101 & $103.4 \%$ & $\left(k_{x}, k_{y}\right)=$ \\
$Z=Z_{0}$ & $\sigma_{y}$ & 0.902 & 7.964 & 3.001 & $100.0 \%$ & $(2.919,0.520)$ \\
$\begin{array}{c}\text { (developable } \\
\text { surface) }\end{array}$ & $\sigma_{x}$ & 0.882 & 7.414 & 3.570 & $119.0 \%$ & $\left(k_{x}, k_{y}\right)=$ \\
\hline
\end{tabular}

Table 4: Stresses $(\mathrm{kN} / \mathrm{m})$ of self-equilibrium shape obtained using correction coefficients from minimal surface and intermediate surface for $Z=0.5 Z_{0}$

\begin{tabular}{c|cccccc}
\hline \multicolumn{1}{c}{$\begin{array}{c}\text { Target curved } \\
\text { surface }\end{array}$} & & $\begin{array}{c}\text { Minimum } \\
\text { value }\end{array}$ & $\begin{array}{c}\text { Maximum } \\
\text { value }\end{array}$ & Mean value & $\begin{array}{c}\text { Mean/ } \\
\text { target }\end{array}$ & $\begin{array}{c}\text { Correction } \\
\text { coefficient }\end{array}$ \\
\hline \multirow{2}{*}{ Minimal surface } & $\sigma_{x}$ & 0.754 & 12.784 & 3.010 & $100.3 \%$ & $\left(k_{x}, k_{y}\right)=$ \\
& $\sigma_{y}$ & 0.299 & 7.851 & 3.013 & $100.4 \%$ & $(1.648,1.048)$ \\
Intermediate & $\sigma_{x}$ & 0.750 & 5.184 & 3.001 & $100.0 \%$ & $\left(k_{x}, k_{y}\right)=$ \\
surface & $\sigma_{y}$ & 0.122 & 10.456 & 3.001 & $100.0 \%$ & $(3.891,0.321)$ \\
\hline
\end{tabular}




\subsection{Comparison of HP surface and developable surface}

In order to confirm the effectiveness of the proposed method, we apply it to an HP surface, which is often used as a target surface of a membrane structure. The surface is defined as

$$
Z=\frac{x y}{A B} H
$$

where $A, B$ and $H$ are defined as shown in Fig. 8, and $A=5 \mathrm{~m}, B=5 \mathrm{~m}$ and $H=2.5 \mathrm{~m}$ in the following example.

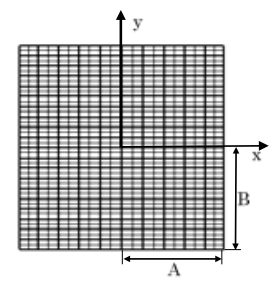

(a)

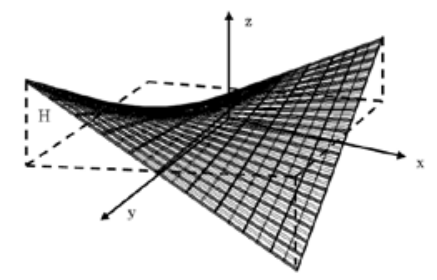

(b)
Figure 8: HP surface: (a) plan view, (b) diagonal view

Note that our purpose is to propose a method for designing the target surface using developable surface. Therefore, we fix the boundary shape and compare the results by solving problems (10) and HP surface, which is commonly used as target surface. The surface is divided into two Bézier surfaces, and the control points of the middle directing curve are considered as design variables. Since it is not possible to have a developable surface for this linear boundary, the objective function in problem (10) is minimized to obtain an approximate developable surface. The cutting patterns are generated as explained in Sec. 3.

Tables 5, 6 and 7 show the stresses obtained from the developable surface, HP surface and intermediate surface. As seen from Table 5, 6 and Table 7 that the mean stress is very close to target stress.

In the following examples, we show that various membrane shapes can be generated using developable surface. Therefore, it is important to note that the developable surface has almost equivalent performance as the HP surface.
Table 5: Stresses $(\mathrm{kN} / \mathrm{m})$ at developable surface

\begin{tabular}{l|lll}
\hline & $\begin{array}{l}\text { Minimum } \\
\text { value }\end{array}$ & $\begin{array}{l}\text { Maximum } \\
\text { value }\end{array}$ & $\begin{array}{l}\text { Mean } \\
\text { value }\end{array}$ \\
\hline$\sigma_{x}$ & 1.764 & 9.123 & 3.003 \\
$\sigma_{y}$ & 0.799 & 9.474 & 3.002 \\
\hline
\end{tabular}

Table 6: Stresses $(\mathrm{kN} / \mathrm{m})$ at HP surface

\begin{tabular}{l|lll}
\hline & $\begin{array}{l}\text { Minimum } \\
\text { value }\end{array}$ & $\begin{array}{l}\text { Maximum } \\
\text { value }\end{array}$ & $\begin{array}{l}\text { Mean } \\
\text { value }\end{array}$ \\
\hline$\sigma_{x}$ & 1.551 & 9.343 & 3.005 \\
$\sigma_{y}$ & 0.757 & 9.184 & 3.003 \\
\hline
\end{tabular}

Table 7: Stresses $(\mathrm{kN} / \mathrm{m})$ at intermediate surface

\begin{tabular}{l|lll}
\hline & $\begin{array}{l}\text { Minimum } \\
\text { value }\end{array}$ & $\begin{array}{l}\text { Maximum } \\
\text { value }\end{array}$ & $\begin{array}{l}\text { Mean } \\
\text { value }\end{array}$ \\
\hline$\sigma_{x}$ & 1.293 & 9.182 & 3.001 \\
$\sigma_{y}$ & 0.729 & 10.375 & 3.001 \\
\hline
\end{tabular}

\section{NUMERICAL EXAMPLES OF DESIGN OF MEMBRANE SURFACES}

The proposed method is applied to simple and connected models as well as a structure combining many simple surfaces.

\subsection{Simple model}

Three types of surfaces are found by solving problems (10), (12) and (13), respectively, from the initial shape in Fig. 9(a). The control points of the two directing curves are denoted by $A_{0}, \ldots, A_{3}$ and $B_{0}, \ldots, B_{3}$, respectively, where the $x$-coordinates of $A_{1}, A_{2}, B_{1}, B_{2}$ and the z-coordinates of $B_{1}, B_{2}$ are chosen as variables. The values of $D^{*}(\mathbf{Q})$ and $\hat{H}(\mathbf{Q})$ of the developable surface are $0.000 \mathrm{~m}^{6}$ and 0.024 $\mathrm{rad}^{2} / \mathrm{m}^{2}$, and $\hat{D}(\mathbf{Q})$ and $H^{*}(\mathbf{Q})$ of the minimal surface are $3.530 \times 10 \mathrm{~m}^{6}$ and $3.772 \times 10^{-3} \mathrm{rad}^{2} / \mathrm{m}^{2}$. The upper-bound value $\bar{H}(\mathbf{Q})$ of the intermediate surface in problem (13) is assigned as $0.014 \mathrm{rad}^{2} / \mathrm{m}^{2}$. 


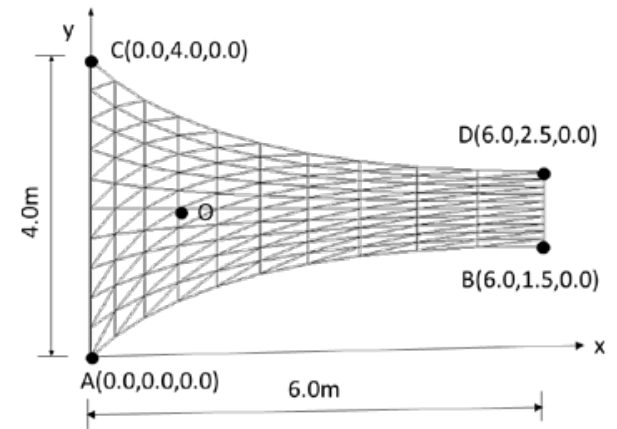

(a)

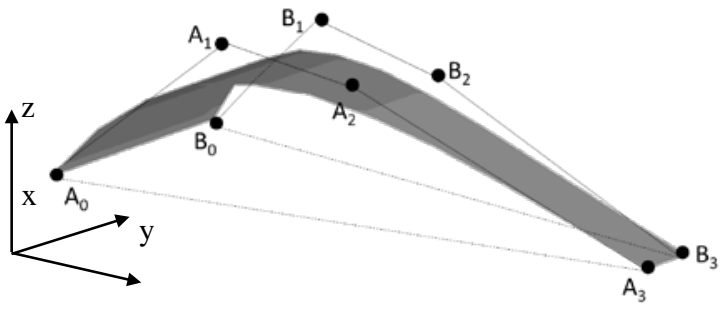

(b)

Figure 9: Initial shape of simple developable surface: (a) plan view, (b) diagonal view.

The obtained target curved surfaces are shown in Figs. 10(a)-(c).

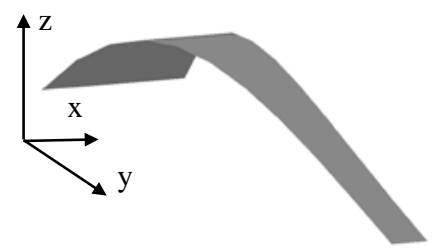

(a)

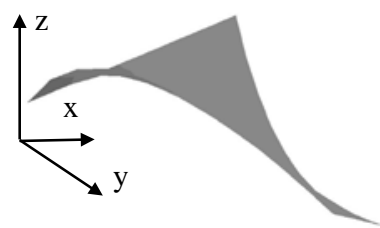

(b)
Cutting patterns are generated by solving problem (14) from three types of target curved surfaces, where the equation of correction (18) is utilized to shrink the cutting sheet. The cutting patterns and final equilibrium shapes of the membrane structures are shown in Figs. 11(a)-(c). The stress values are listed in Table 8, which shows that the mean values of the intermediate surface are close to the target values if the correction coefficients $k_{x}$ and $k_{y}$ are assigned appropriately. It is also noted that the intermediate surface has the larger minimum and smaller maximum stresses than the other two cases. The obtained membrane structure has a slight difference with the shape of the target curved surface. The values of $\Delta Z$ measured at point $\mathrm{O}$ indicated in Fig. 9(a) are $0.083 \mathrm{~m}, 0.019 \mathrm{~m}$ and $0.093 \mathrm{~m}$, respectively, for developable surface, minimal surface and intermediate surface. Those values are $0.025 \mathrm{~m}, 0.015 \mathrm{~m}$ and $0.037 \mathrm{~m}$ if Eq. (17) is used. A complex shape can be designed combining simple shapes. Fig. 12 is stress diagram. Fig. 13 shows a membrane structure obtained by combining 13 simple surfaces in Fig. 11(a).

Figure 10: Diagonal view of target curved surfaces: (a) developable surface, (b) minimal surface, (c) intermediate
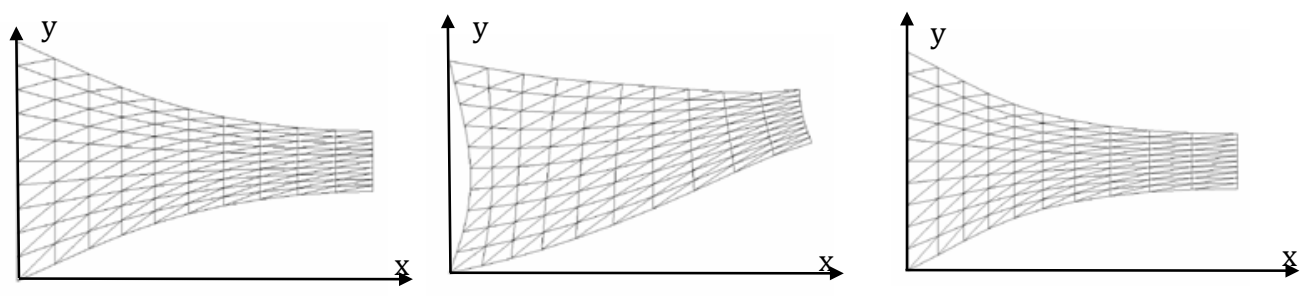

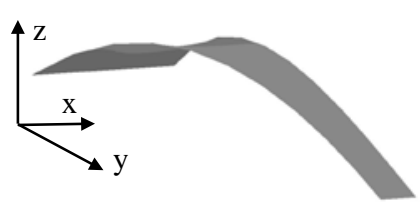

(a)

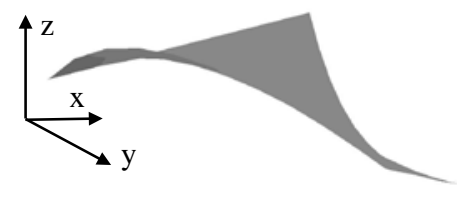

(b)
(c)
gattern and self-equilibrium shape from three types of target curved surface:

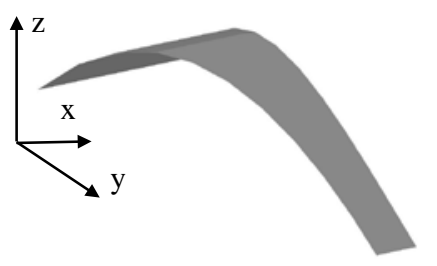

(c)

\section{surface between developable and minimal surfaces}

Figure 11: Cutting pattern and self-equilibrium shape from three types of target curved surface: (a) developable surface, (b) minimal surface, (c) intermediate surface between developable and minimal surfaces 


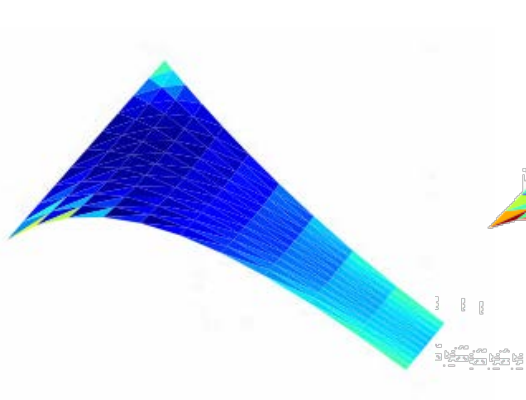

(a)

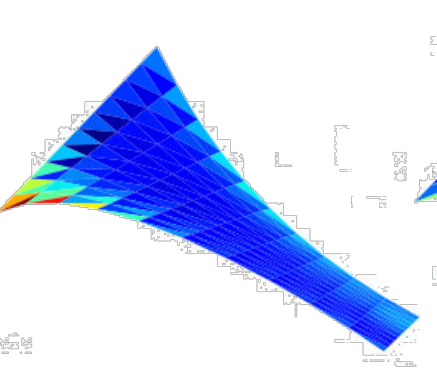

(b)

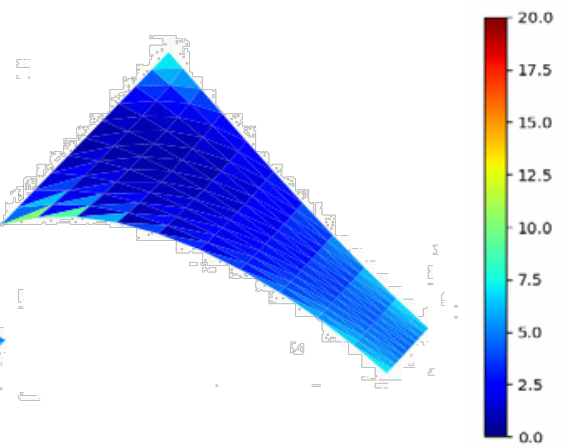

(c)

Figure 12: Stress view: (a) developable surface, (b) minimal surface, (c) intermediate surface between developable and minimal surfaces

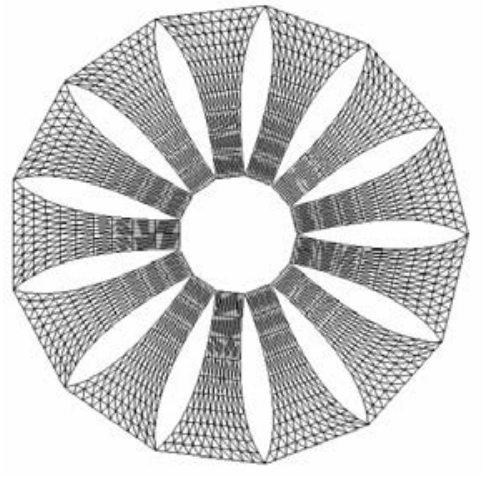

(a)

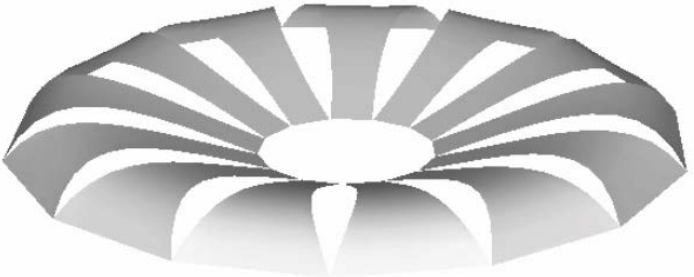

(b)

Figure 13: Combined membrane structure: (a) plan view, (b) diagonal view

Table 8: Stresses $(\mathrm{kN} / \mathrm{m})$ of self-equilibrium shape of simple model

\begin{tabular}{ccccccc}
\hline $\begin{array}{c}\text { Target curved } \\
\text { surface type }\end{array}$ & $\begin{array}{c}\text { Stress } \\
\text { type }\end{array}$ & $\begin{array}{c}\text { Stress } \\
\text { minimum } \\
\text { value }\end{array}$ & $\begin{array}{c}\text { Stress } \\
\text { maximum } \\
\text { value }\end{array}$ & $\begin{array}{c}\text { Stress } \\
\text { mean } \\
\text { value }\end{array}$ & $\begin{array}{c}\text { Mean/ } \\
\text { target }\end{array}$ & $\begin{array}{c}\text { Correction } \\
\text { coefficient }\end{array}$ \\
\hline Developable & $\sigma_{x}$ & 1.478 & 5.795 & 3.328 & $110.9 \%$ & $\left(k_{x}, k_{y}\right)=$ \\
surface & $\sigma_{y}$ & 0.003 & 12.482 & 3.551 & $118.4 \%$ & $(3.44,0.70)$ \\
Minimal & $\sigma_{x}$ & 0.012 & 19.210 & 4.751 & $158.4 \%$ & $\left(k_{x}, k_{y}\right)=$ \\
surface & $\sigma_{y}$ & 0.124 & 19.786 & 3.887 & $129.6 \%$ & $(1.39,1.794)$ \\
Intermediate & $\sigma_{x}$ & 1.378 & 5.019 & 3.004 & $100.1 \%$ & $\left(k_{x}, k_{y}\right)=$ \\
surface & $\sigma_{y}$ & 0.653 & 10.080 & 3.089 & $103.0 \%$ & $(2.819,0.582)$ \\
\hline
\end{tabular}

\subsection{Connected model}

The initial shape of surface for solving optimization problems (10), (12) and (13) is assigned as shown in Fig. 14 on a $6.0 \times 4.0 \mathrm{~m}$ rectangular region. The surface is supported at nine points $A, B, C, D, E, F$, $G, H, I$ as shown in Fig. 14(a). The $x$-coordinates of $A_{1}, A_{2}, A_{4}, A_{5}, B_{1}, B_{2}, B_{4}, B_{5}, C_{1}, C_{2}, C_{4}$,
$C_{5}$ and the z-coordinates of $A_{0}, A_{1}, A_{2}, A_{4}, A_{5}$, $A_{6}, B_{0}, B_{1}, B_{2}, B_{4}, B_{5}, B_{6}, C_{0}, C_{1}, C_{2}, C_{4}$, $C_{5}, C_{6}$ are chosen as variables in problems (10) and (13) for the developable surface and the intermediate surface, while the points $B_{1}, B_{2}, B_{3}, B_{4}, B_{5}$ are chosen for the variables of $z$-coordinates in problem (12) for the minimal surface. 
By allowing variation of locations of the supports except for the four corners, it is easy to reduce the norm of mean curvature, while satisfying the continuity conditions. The curved surface is composed of four Bézier surfaces (dashed line part in Fig. 14(a)), and the directing curves are represented by six Bézier curves.

The values of $D^{*}(\mathbf{Q})$ and $\hat{H}(\mathbf{Q})$ are $0.000 \mathrm{~m}^{6}$ and $0.774 \mathrm{rad}^{2} / \mathrm{m}^{2}$, and $\hat{D}(\mathbf{Q})$ and $H^{*}(\mathbf{Q})$ are $16.20 \mathrm{~m}^{6}$ and $0.393 \mathrm{rad}^{2} / \mathrm{m}^{2}$. The value of $\hat{H}(\mathbf{Q})$ in problem (13) for obtaining an intermediate surface is 0.631 $\mathrm{rad}^{2} / \mathrm{m}^{2}$. The $G^{0}$ continuity is assigned along the internal boundaries DE and DF, while $G^{1}$ continuity is assigned along lines $\mathrm{BE}$ and $\mathrm{EH}$. The target curved surfaces obtained by solving problems (10), (12) and (13) are shown in Fig. 15

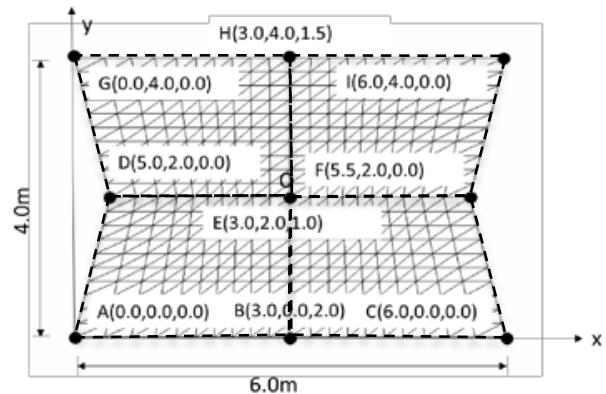

(a)

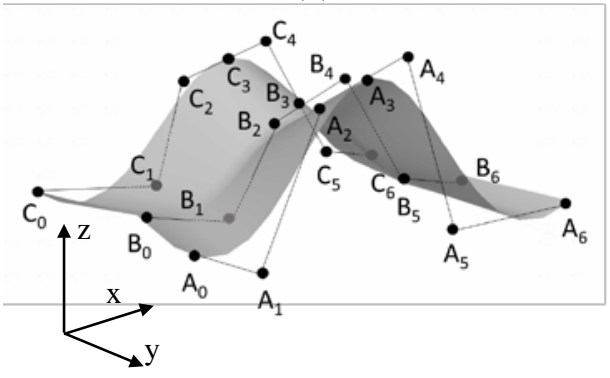

(b)

Figure 14: Initial shape of connected developable surfaces: (a) plan view, (b) diagonal view

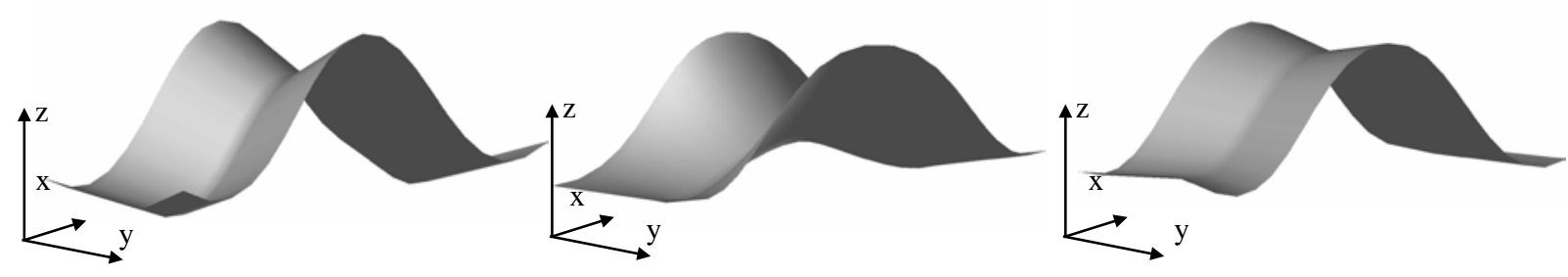

(a)

(b)

(c)

Figure 15: Diagonal view of target curved surfaces: (a) developable surface, (b) minimal surface, (c) intermediate surface between developable and minimal surfaces
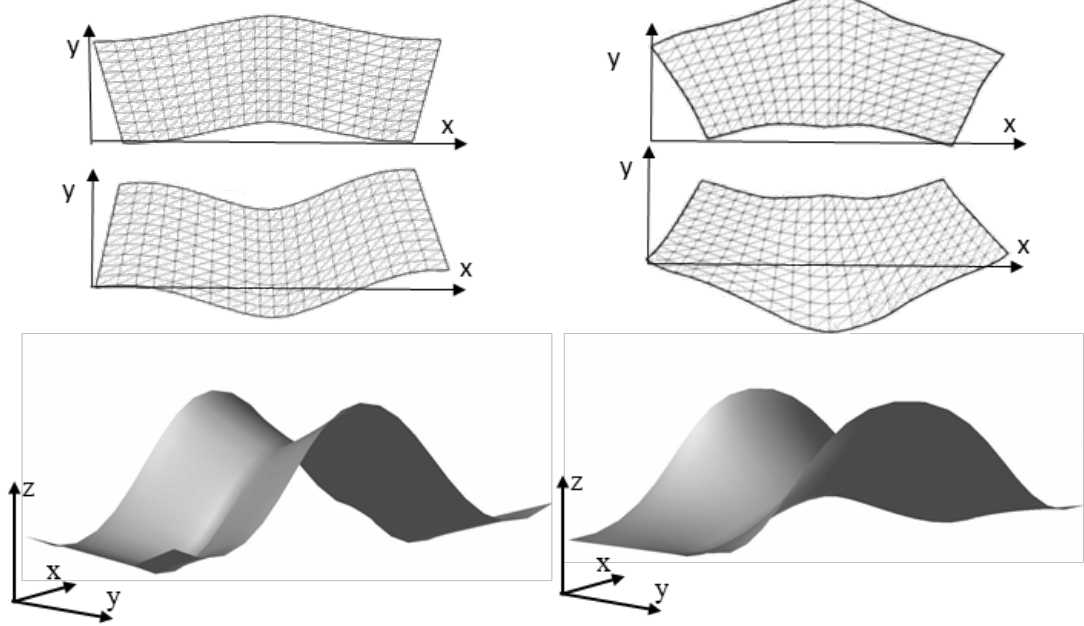

(a)

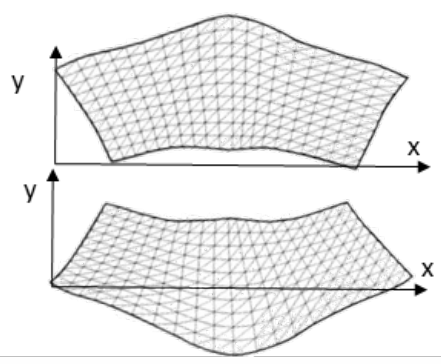

(b)
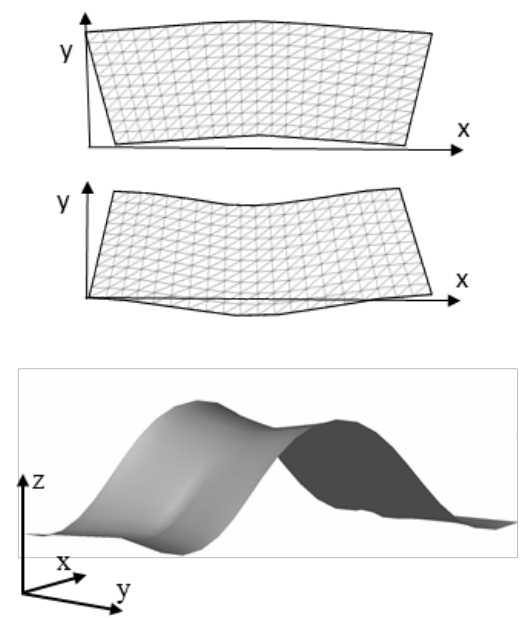

(c)

Figure 16: Plan view and diagonal view of self-equilibrium shape obtained from three different target curved surface: (a) developable surface, (b) minimal surface, (c) intermediate surface between developable and minimal surfaces 
Vol. 59 (2018) No. 3 September n. 197

Table 9: Stresses $(\mathrm{kN} / \mathrm{m})$ of self-equilibrium shape of complex model

\begin{tabular}{c|cccccc}
\hline $\begin{array}{c}\text { Target curved } \\
\text { surface type }\end{array}$ & $\begin{array}{c}\text { Stress } \\
\text { type }\end{array}$ & $\begin{array}{c}\text { Stress } \\
\text { minimum } \\
\text { value }\end{array}$ & $\begin{array}{c}\text { Stress } \\
\text { maximum } \\
\text { value }\end{array}$ & $\begin{array}{c}\text { Stress } \\
\text { mean value }\end{array}$ & $\begin{array}{c}\text { Mean/ } \\
\text { target }\end{array}$ & $\begin{array}{c}\text { Correction } \\
\text { coefficient }\end{array}$ \\
\hline $\begin{array}{c}\text { Developable } \\
\text { surface }\end{array}$ & $\sigma_{x}$ & 1.075 & 8.904 & 3.862 & $128.7 \%$ & $\left(k_{x}, k_{y}\right)=$ \\
& $\sigma_{y}$ & 0.096 & 13.367 & 3.052 & $101.7 \%$ & $(3.875,1.280)$ \\
Minimal & $\sigma_{x}$ & 0.011 & 13.054 & 4.068 & $135.6 \%$ & $\left(k_{x}, k_{y}\right)=$ \\
surface & $\sigma_{y}$ & 0.043 & 13.463 & 3.794 & $126.5 \%$ & $(3.029,1.374)$ \\
& $\sigma_{x}$ & 0.068 & 9.641 & 3.502 & $116.7 \%$ & $\left(k_{x}, k_{y}\right)=$ \\
Intermediate & $\sigma_{y}$ & 0.070 & 10.636 & 3.006 & $100.2 \%$ & $(3.735,0.985)$ \\
\hline
\end{tabular}

Since the boundary frame including the internal boundary EDF in Fig. 14(a) are assumed to be rigid, the target curved surface is divided into the front part and the back part with respect to EDF. The correction coefficients are assigned as listed in Table 9 to obtain cutting patterns as shown in Figs. 16(a), (b), (c) for the developable surface, minimal surface and intermediate surface, respectively. Finally, by minimization of the strain energy in the front and back parts of the target curved surface, respectively, the self-equilibrium shapes are obtained as shown in the bottom figures of Fig. 16(a), (b), (c). The minimum and maximum stress values in table 9 are respectively the minimum and maximum stress values in the two segmented surfaces in Fig. 16(a), (b), (c).

Table 9 shows the stress state and the correction coefficients of the obtained membrane structure. As seen from the table, the mean stress is approximately coincident with the target value, and the intermediate surface has the best values. However, the minimum value and the maximum value are considerably far from the target value. Due to the complexity of the boundary of structure, the obtained target curved surfaces are quite different with each other, difference between the cutting pattern and the plan shape of target curved surface is also very large. Therefore, it is difficult to determine the appropriate value of correction coefficient for obtaining the cutting pattern. The obtained membrane structure has a slight difference with the shape of the target curved surface. The values of $\Delta Z$ measured at point $\mathrm{O}$ indicated in Fig. 14(a) is $0.048 \mathrm{~m}, 0.054 \mathrm{~m}$ and $0.054 \mathrm{~m}$, respectively, for developable surface, minimal surface and intermediate surface. Those values are $0.017 \mathrm{~m}, 0.021 \mathrm{~m}$ and $0.019 \mathrm{~m}$ if Eq. (17) is used.

\section{CONCLUSION}

A design method has been presented for curved membrane surfaces. In the proposed method, an intermediate solution between minimal surface and developable surface is generated by solving a multiobjective optimization problem using a constraint method. The surface is modeled by $(1, n)$-Bézier surface representing ruled surface with Bézier curve as generating curves. Developability conditions are assigned to a ruled surface to obtain a developable surface through optimization, where the variables are the coordinates of the control points of Bézier curves.

The target curved surface is triangulated and projected to a plane, and the norm of difference in edge lengths of triangular elements between those on surface and plane are minimized. A cutting pattern is determined by reducing the strain corresponding to the target stress to this developed surface. A simple approach of using correction coefficients has been proposed. The equilibrium shape is generated and its stress distribution is investigated by minimizing strain energy computed due to forced deformation of the cutting sheet to the boundary of the curved surface.

By using the proposed method, the cutting patterns of membrane structures can be obtained through a simple single step process of developing the curved surface to a plane and reducing the deformation due to the target stress. Although the minimal surface, which can be at equilibrium in uniform tension state, is usually used as the target curved surface of a membrane structure, a developable surface may be another candidate, because the curved surface is generated by connecting plane cutting sheets. In this respect, the best surface may be an intermediate surface between the minimal and developable surfaces. 
It has been shown in the example of HP model that developable surface and intermediate surface can be effectively used as target surfaces of membrane structures. These surfaces have almost equivalent performance as the minimal surface. However, the intermediate surface has the best performance in both of the simple and connected models in Sec. 5.

It should be noted that the surface shapes of three types are very different; therefore, the proposed method can be effectively used for generating various complex shapes of membrane structures connecting the surfaces using $G^{0}$ and $G^{1}$ continuity conditions. It has also been shown that the difference between the target curved surface and the equilibrium surface generated from cutting patterns are moderately small.

Each unit is generated from a single cutting sheet to investigate the extreme case, although it is obvious that the stress distribution is improved by using several cutting sheets for each surface.

\section{ACKNOWLEDGEMENT}

This study is partially supported by JSPS KAKENHI No. 16K14338.

\section{REFERENCES}

[1] Bletzinger $K_{.}-U_{\text {., }} \quad \boldsymbol{R a m m} \boldsymbol{E}$., Structural optimization and form finding of light weight structures. Comp Struct 2001:79:2053-2062. (DOI: 10.1016/S0045-7949(01)00052-9)

[2] Beatini V, Royer-Carfagni G. Soap film analogy for anisotropically stretched membranes and cable nets. Struct. Multidisc. Optim. 2017:55:885-898.

(DOI: 10.1007/s00158-016-1533-z)

[3] Bletzinger KU, Wüchner R, Daoud $F$, Camprubí $N$. Computational methods for form finding and optimization of shells and membranes. Comp. Meth. Appl. Mech. Engrg. 2005:194:3438-3452.

(DOI: 10.1016/j.cma.2004.12.026)

[4] Farin G. Curves and Surfaces for Computer Aided Geometric Design. Academic Press 1997.

[5] Kumar GCCR, Srinivasan P, Holla VD, Shasty KG, Prakash BG. Geodesic curve computations on surfaces. Computer Aided
Geometric Des. 2003:20:119-133. (DOI: 10.1016/S0167-8396(03)00023-2)

[6] Ishii $\boldsymbol{K}$. Form finding analysis in consideration of cutting patterns of membrane structures, Int. J. Space Struct. 1999:14(2):105-119.

(DOI: 10.1260/0266351991494731)

[7] Meek JL, Tan KY. Post-form finding determination of geodesic lines in cutting pattern design for membrane structures. Int. J. Space Struct. 1986:2:231-239. (DOI: $10.1177 / 026635118700200405)$

[8] Punurai W, Tongpool W, Morales JH., Implementation of genetic algorithm for optimum cutting pattern generation of wrinkle free finishing membrane structures. Finite Element Analysis and Design 2012:58:84-90. (DOI: 10.1016/j.finel.2012.04.008)

[9] Gale S, Lewis WJ. Patterning of tensile fabric structures with a discrete element model using dynamic relaxation. Comp Struct 2016:169:112-121.

(DOI: 10.1016/j.compstruc.2016.03.005)

[10] Barnes MR. Form-finding and analysis of prestressed nets and membranes. Comp Struct. 1988:30:685-695. (DOI: 10.1016/00457949(88)90304-5)

[11] Lu K, Accorsi M. Leonard J. Finite element analysis of membrane wrinkling. Int. J. Numer Meth Eng 2001:50:1017-1038. (DOI: 10.1002/10970207(20010220)50:5<1017::AIDNME47>3.0.CO;2-2)

[12] X. Xia, J.L. Meek. Computer cutting pattern generation of membrane structures International Journal of space structures Volume 15, Issue 2, Pages 95-110. (DOI: 10.1260/0266351001495008)

[13] Tsubota H, Yosida A, Kurokawa $\boldsymbol{Y}$. Theoretical analysis of actual initial equilibrium state for membrane structures based on cutting patterns. J Struct Constr Eng 1987:373:101-110. (in Japanese)

[14] Ohsaki M, Uetani K, Takatani S. Shapestress trade-off design method of membrane structures by using inverse problem approach. 
J Struct Constr Eng 1996:488:107-115. (in Japanese) (DOI: 10.3130/aijs.61.107_3)

[15] Ohsaki M, Fujiwara J. Developability conditions for prestress optimization of a curved surface. Comp Meth Appl Mech Engrg 2003:192:77-94. (DOI: 10.1016/S00457825(02)00523-6)

[16] S. Shon, S. Lee, K. Lee. Smooth cutting pattern generation technique for membrane structures using geodesic line on subplane and spline interpolation. J. Central South Univ. 2013:20(11):3131-3141.

(DOI: 10.1007/s11771-013-1836-9)

[17] Moncrieff E., Topping BHV. Computer methods for the generation of membrane cutting patterns Comp. Struct. 1990:37(4):441-450. (DOI: 10.1016/00457949(90)90034-Y)

[18] Sánchez-Reyes $\boldsymbol{J}$. On the construction of minimal surfaces from geodesics. Applied Mathematical Modelling 2016:40:1676-1682. (DOI: 10.1016/j.apm.2015.07.011)

[19] Yokosuka Y, Honma T. Form-finding analysis for membrane structures based on discrete differential geometry: Verification of unit stress distribution, Proc. Int. Assoc. for Shell and Spatial Struct. Annual Symposium (IASS2017), Paper No. 9560, 2017.

[20] Gosling PD, Lewis WJ. Optimal structural membranes II: Form-finding o prestressed membranes using a curved quadrilateral finite element for surface definition. Comp. Struct. 1996:61(5):885-895. (DOI: 10.1016/00457949(96)00091-0)

[21] Aumann G. A simple algorithm for designing developable Bézier surfaces. Computer Aided Geometric Des 2003:20:601-619. (DOI: 10.1016/j.cagd.2003.07.001)

[22] Shuxun W, Zhengli YE. Design of developable Bézier surfaces by de Casteljau algorithm. Computer Eng Appl 2007:43(23):21-23.

[23] Cui J, Ohsaki M, Nakamura K. Shape optimization of free-form shells consisting of developable surfaces. J Struct Constr Eng 2016:737:1137-1143. (in Japanese)
[24] Nakamura K, Ohsaki M, Cui J. Shape optimization of free-form shells consisting of developable surfaces. Proc IASS Symposium 2016, Tokyo, Int Assoc Shell and Spatial Struct 2016: Paper No. CS2E-1057.

[25] Aumann G. Degree elevation and developable Bézier surface. Computer-Aided Geometric Des 2004:21:661-670.

(DOI: 10.1016/j.cagd.2004.04.007)

[26] Chu CH, Séquin $\boldsymbol{C H}$. Developable Bézier patches: Properties and design. ComputerAided Des 2002:34:511-527. (DOI: 10.1016/S0010-4485(01)00122-1)

[27] Dongren C, Guojin W. Developable Bézier parametric surfaces. J. Computer Aided Design \& Computer Graphics 2003:15(5):570-575.

[28] Monterde J. Bézier surfaces of minimal area: The Dirichlet approach. Comp. Aided Geometric Des. 2004:21:117-136. (DOI: 10.1016/j.cagd.2003.07.009)

[29] Koohestani $\boldsymbol{K}$. Nonlinear force density method for the form-finding of minimal surface membrane structures. Communications in Nonlinear Science and Numerical Simulation 2014:19:2071-2087. (DOI: 10.1016/j.cnsns.2013.10.023)

[30] Fujita S, Ohsaki M. Shape optimization of free-form shells using invariants of parametric surface. Int J Space Struct 2010:25:143-157. (DOI: 10.1260/0266-3511.25.3.143)

[31] Ohsaki M, Saburi S, Takeda F. Approximate method for cutting pattern optimization of membrane structures. Proc IASS 2017, Hamburg, Germany, 2017.

[32] Ohsaki M, Fujiwara J, Takeda F. Approximate method for cutting pattern optimization of frame-supported and pneumatic membrane structures. http://arxiv.org/abs/1712.04598.

[33] Bouzidi R, Le Van A. Numerical solution of hyperelastic membranes by energy minimization. Comp Struct 2004:82:19611969.

(DOI: 10.1016/j.compstruc.2004.03.057) 